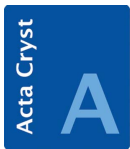

FOUNDATIONS

ADVANCES

ISSN 2053-2733

Received 25 June 2015

Accepted 26 June 2015

Keywords: MicroED; nanocrystals; electron diffraction.

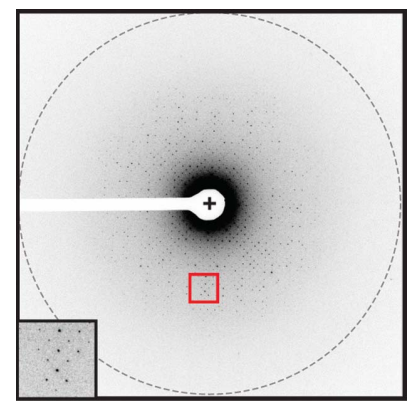

C 2015 International Union of Crystallography

\section{Accessible atomic structures from sub-micron protein crystals}

\author{
Jose A. Rodriguez
}

Department of Biological Chemistry, UCLA-DOE Institute for Genomics and Proteomics, University of California, Los Angeles, Los Angeles, California, USA

New instruments and techniques expand the reach of crystallography, making it more efficient and/or accessible to the greater scientific community. However, despite technological improvements, crystallography still faces outstanding challenges. As an example, structure determination from sub-micron crystals remains largely out of reach for conventional crystallography. In this issue of Acta Crystallographica Section A, the lead article by Hattne et al. (2015) outlines the steps involved in the collection and processing of data from thin (i.e. sub-micron thick) crystals by the three-dimensional electron crystallography method known as MicroED

A crystallographer can use table-top or synchrotron X-ray sources to collect diffraction data from protein crystals that are microns in size or larger. These sources are constantly improving, but are not yet advanced enough for use with sub-micron protein crystals (Holton \& Frankel, 2010). Smaller crystals require the use of brighter X-ray sources (Gruner \& Lattman, 2015). X-ray free-electron lasers (XFELs) have helped to produce structures from nanocrystals, including an experimentally phased structure of lysozyme (Barends et al., 2014). In principle, using a bright, coherent X-ray beam and new approaches to phasing, the tiniest crystals could be used (Miao \& Rodriguez, 2014) and even single protein molecules could be resolved (Miao et al., 2015). In practice, however, XFEL crystallography faces obstacles. XFELs are expensive to build, can require large quantities of sample (Boutet et al., 2012; Cohen et al., 2014), and with only two sources currently available worldwide (Emma et al., 2010; Ishikawa et al., 2012), beam time is scarce.

Two years ago, a group led by Tamir Gonen determined the structure of lysozyme at $2.9 \AA$ resolution from thin, sub-micron-sized crystals at cryogenic temperatures using an electron microscope (Shi et al., 2013). A low-dose tilt series allowed them to extend electron crystallography to three dimensions. They coined the term 'MicroED' for their method. Since that initial demonstration, several other structures have been determined by MicroED. All have relied on molecular replacement for structure determination. Shortly after the first demonstration of MicroED, the Gonen group developed a method of continuous rotation to improve the quality of the measured MicroED intensities, once again on lysozyme crystals (Nannenga, Shi, Leslie \& Gonen, 2014). Two more structures were then determined; catalase (Nannenga, Shi, Hattne et al., 2014; Yonekura et al., 2015) and calcium ATPase (Yonekura et al., 2015). Over the course of two years, use of the method has spread to multiple laboratories around the world that are equipped with different electron microscopes and detectors.

The use of an electron microscope for this purpose is not entirely surprising. Electrons are the quanta of choice for structure determination of proteins using single-particle methods: they offer a larger scattering cross section than X-rays and can be precisely manipulated by electromagnetic lenses. Modern electron microscopes are engineered to visualize even single protein molecules with high spatial resolution (Cheng et al., 2015). Since the seminal studies on bacteriorhodopsin by Henderson and colleagues (Henderson et al., 1990), electrons have also been used to solve structures of proteins from two-dimensional crystals, recently at atomic resolution (Gonen et al., 2005).

The method of MicroED is at heart a marriage of electron cryo-microscopy (cryo-EM) and crystallography. Crystal growth and screening for MicroED is adapted from standard crystallographic approaches (vapor diffusion and batch), using similar screens and equipment. EM grids are prepared by treating crystals in solution like a single-particle suspension, a technique borrowed from protocols well known to cryo-EM specialists. 


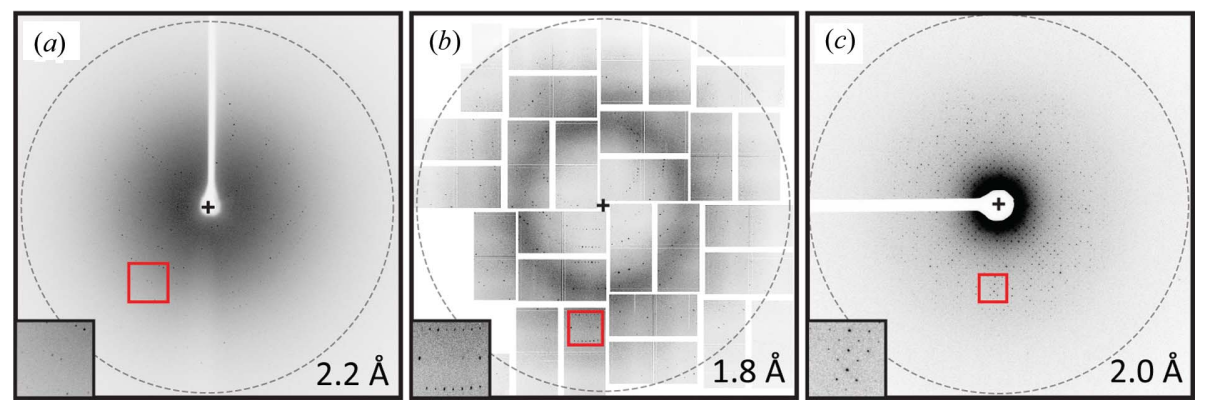

Figure 1

Diffraction micrographs from (a) a lysozyme crystal, tens of microns large, collected using a rotating-anode X-ray source, (b) a lysozyme nanocrystal, collected using an XFEL source (data publicly available from the Coherent X-ray Imaging Data Bank at http://cxidb.org/id-17.html), and (c) a submicron-sized lysozyme crystal, collected by MicroED. Insets show magnified areas from each of the patterns. Rings mark the resolution near the edge of each pattern. Image $(a)$ courtesy of D. Cascio and M. Collazo, and images $(b)$ and $(c)$ courtesy of J. Hattne.

Likewise, data collection is conducted as a cryo-EM experiment on a modern electron microscope, albeit in diffraction mode, a protocol accessible to versed microscopists. Electrondiffraction patterns are collected as images or movies during unidirectional rotation of crystals and converted by standalone software into diffraction micrographs analogous to the oscillation photographs collected by X-ray crystallographers (Fig. 1). Two crystallography software packages are now capable of natively processing micrographs from MicroED experiments: MOSFLM (Leslie \& Powell, 2007) and XDS (Kabsch, 2010); a third has been demonstrated but is not currently accessible (Yonekura et al., 2015). The reduced data can then progress through standard crystallography workflows for molecular replacement, model building and refinement (Adams et al., 2010; Murshudov et al., 2011).

Challenges remain for MicroED. Opportunities for improvement of the method begin with innovations to highthroughput screening of potential crystals. Currently, screening relies on visual inspection followed by observation using an electron microscope. To maximize the likelihood that crystals diffract, optimization and standardization of handling protocols for crystals in various mother liquors is required. This preserves the character of a crystal against plunge freezing on a solid support followed by exposure to vacuum. At large tilt angles, greater than $70^{\circ}$, the grid can obstruct the beam and prevent a small cone of data from being collected. Merging data from multiple crystals can obviate this effect and complete data sets are acquired provided that the crystals do not strictly adopt a preferred orientation on the grid. As with conventional X-ray crystallography, radiation damage ultimately limits data collection, despite cryogenic preservation and the very low electron dose required for MicroED. Lastly, and perhaps most importantly, phasing stands as an important milestone for MicroED. New structures could be generated using MicroED by molecular replacement (Rossmann \& Blow, 1962). However, the widespread implementation of MicroED may require a new phasing solution for electron diffraction. Although unproven for electrons, adoption of phasing techniques developed for X-ray crystallography such as isomorphous replacement (Perutz, 1956) may be possible (Burmester \& Schröder, 1997).
MicroED follows in the wake of other successful innovations to crystallography. By enlisting the use of more affordable and accessible instruments, Hattne et al. invite the microscopist and crystallographer alike to solve atomic structures from protein nanocrystals using MicroED.

\section{References}

Adams, P. D. et al. (2010). Acta Cryst. D66, 213-221.

Barends, T. R. M., Foucar, L., Botha, S., Doak, R. B., Shoeman, R. L., Nass, K., Koglin, J. E., Williams, G. J., Boutet, S., Messerschmidt, M. \& Schlichting, I. (2014). Nature, 505, 244-247.

Boutet, S. et al. (2012). Science, 337, 362-364.

Burmester, C. \& Schröder, R. R. (1997). Scan. Microsc. 11, 323-334.

Cheng, Y., Grigorieff, N., Penczek, P. A. \& Walz, T. (2015). Cell, 161, 438-449.

Cohen, A. E. et al. (2014). Proc. Natl Acad. Sci. USA, 111, 1712217127.

Emma, P. et al. (2010). Nat. Photon. 4, 641-647.

Gonen, T., Cheng, Y., Sliz, P., Hiroaki, Y., Fujiyoshi, Y., Harrison, S. C. \& Walz, T. (2005). Nature, 438, 633-638.

Gruner, S. M. \& Lattman, E. E. (2015). Annu. Rev. Biophys. 44, 33-51.

Hattne, J., Reyes, F. E., Nannenga, B. L., Shi, D., de la Cruz, J. M., Leslie, A. G. W. \& Gonen, T. (2015). Acta Cryst. A71, 353-360.

Henderson, R., Baldwin, J. M., Ceska, T. A., Zemlin, F., Beckmann, E. \& Downing, K. H. (1990). J. Mol. Biol. 213, 899-929.

Holton, J. M. \& Frankel, K. A. (2010). Acta Cryst. D66, 393-408.

Ishikawa, T. et al. (2012). Nat. Photon. 6, 540-544.

Kabsch, W. (2010). Acta Cryst. D66, 125-132.

Leslie, A. G. W. \& Powell, H. R. (2007). In Evolving Methods for Macromolecular Crystallography, edited by R. J. Read \& J. L. Sussman, pp. 41-51. Dordrecht: Springer.

Miao, J., Ishikawa, T., Robinson, I. K. \& Murnane, M. M. (2015). Science, 348, 530-535.

Miao, J. \& Rodriguez, J. A. (2014). IUCrJ, 1, 3-4.

Murshudov, G. N., Skubák, P., Lebedev, A. A., Pannu, N. S., Steiner, R. A., Nicholls, R. A., Winn, M. D., Long, F. \& Vagin, A. A. (2011). Acta Cryst. D67, 355-367.

Nannenga, B. L., Shi, D., Hattne, J., Reyes, F. E. \& Gonen, T. (2014). eLife, $\mathbf{3}, \mathrm{e} 03600$.

Nannenga, B. L., Shi, D., Leslie, A. G. W. \& Gonen, T. (2014). Nat. Methods, 11, 927-930.

Perutz, M. F. (1956). Acta Cryst. 9, 867-873.

Rossmann, M. G. \& Blow, D. M. (1962). Acta Cryst. 15, 24-31.

Shi, D., Nannenga, B. L., Iadanza, M. G. \& Gonen, T. (2013). eLife, 2 , e01345

Yonekura, K., Kato, K., Ogasawara, M., Tomita, M. \& Toyoshima, C. (2015). Proc. Natl Acad. Sci. USA, 112, 3368-3373. 